\title{
Pueratin improves diminished ovarian reserve by inhibiting apoptosis
}

\author{
QUAN QI, XIQIAN ZHANG, LI YAO, YE CHEN and HUINAN WENG \\ Reproductive Medicine Center, Guangdong Women and Children Hospital, \\ Guangdong, Guangzhou 511442, P.R. China
}

Received June 7, 2021; Accepted July 30, 2021

DOI: $10.3892 /$ etm.2021.10858

\begin{abstract}
Pueratin (Pue) is an extract from Pueraria lobata, and exhibits therapeutic effects for the treatment of inflammation. However, the beneficial effects and mechanisms underlying Pue in the treatment of diminished ovarian reserve (DOR) remains to be fully elucidated. The aim of the present study was to investigate the effect of Pue on Bcl-2 and Bax protein expression in rats with DOR, associated with infertility within clinical practice, induced by 4-vinylcyclohexene diepoxide (VCD). A model of DOR was established in female Sprague Dawley rats by an intraperitoneal injection of $80 \mathrm{mg} / \mathrm{kg}$ VCD daily for 45 days. From day 1, the Sprague Dawley rats were orally administered with drugs daily for 45 days. They were divided into normal, model, Pue-low dose (L), Pue-medium dose (M) and Pue-high dose (H) groups (50, 100 and $300 \mathrm{mg} / \mathrm{kg}$ Pue, respectively). Follicle-stimulating hormone $(\mathrm{FSH})$, luteinizing hormone $(\mathrm{LH})$ and estradiol $\left(\mathrm{E}_{2}\right)$ levels were subsequently detected using ELISA. H\&E staining and TUNEL staining were used to evaluate histopathological changes and apoptosis levels in the ovary, respectively. Bcl-2 and Bax protein expression levels in rat ovaries were evaluated using immunohistochemistry and western blotting. Compared with those in the model group, FSH and LH levels in the Pue-L, -M and - $\mathrm{H}$ groups were significantly decreased, whilst $\mathrm{E}_{2}$ levels were significantly increased $(\mathrm{P}<0.05)$. After intragastric administration, the volume of the ovaries and uteri of rats in the Pue groups was increased compared with the model group, and the numbers of primordial follicles and primary follicles were also increased. The number of apoptotic cells and the expression of Bax were significantly reduced in a dose-dependent manner $(\mathrm{P}<0.05)$, compared with the model group. In addition, $\mathrm{Bcl}-2$ protein expression and the $\mathrm{Bcl}-2 / \mathrm{Bax}$ ratio were found to be significantly increased in
\end{abstract}

Correspondence to: Dr Huinan Weng, Reproductive Medicine Center, Guangdong Women and Children Hospital, 521 Xingnan Dadao, Guangdong, Guangzhou 511442, P.R. China

E-mail: boyweng0429@21cn.com

Key words: pueratin, diminished ovarian reserve, cell apoptosis, Bcl-2, Bax the Pue-treated groups in a dose-dependent manner $(\mathrm{P}<0.05)$, compared with the model group. In conclusion, Pue treatment improved ovarian function by regulating hormone balance in addition to Bcl-2 and Bax expression.

\section{Introduction}

Diminished ovarian reserve (DOR) is a condition that reduces the quantity and/or quality of ovarian follicles before the age of 40 as a result of ovarian dysfunction. Ovarian dysfunction is a result of various factors, such as endocrine disorders and ovarian injury, which lead to a decline in fertility (1). Due to DOR, an increasing number of young women experience premature oligomenorrhea, amenorrhea, infertility or miscarriage, which greatly reduces the success rate of assisted reproductive technology used for infertility (2). If not treated in a timely manner, this condition can develop into premature ovarian failure (3). The most commonly used treatment method in modern medicine is hormone replacement therapy (HRT), which involves the supplement of estrogen (4) and the use of ovulation stimulators. Medications used to stimulate ovarian function in clinical practice include letrozole, clomiphene citrate, gonadotropins and pulsatile gonadotropin releasing hormone (5). Despite this therapeutic option being available, not only is the morbidity as a result of DOR increasing annually and the efficacy of HRT not improving, potential risks associated with the development of breast cancer, endometrial cancer and heart disease are also increased (6-9).

Pueratin (Pue) is a flavonoid that can be extracted from the perennial vine Pueraria lobata with various reported pharmacological activities, including antioxidant, anti-inflammatory, anti-apoptotic, neuroprotective and cardioprotective properties (10). It has been previously demonstrated to exert effective protection in several types of cell injury, including neurons, epithelial cells, vascular endothelial cells and cardiomyocytes (11-14). However, the potential effect of Pue on DOR remains unclear. Previous studies have suggested that Bcl-2 and Bax are associated with apoptosis in mammalian cells $(15,16)$. A previous study (7) found that Pue exerted therapeutic effects against ovarian failure via regulation of the Wnt/ $\beta$-catenin signaling pathway and oxidative stress. However, since the concentration of Pue was low and the effects of Pue on follicular stimulating hormone (FSH) levels in the rats with DOR remain unknown (4). 
Therefore, in the present study, a physiological DOR rat model was established by vinyl-cyclohexene-dioxide (VCD) injection to assess the effects of Pue on the expression levels of the apoptosis-related proteins, Bcl-2 and Bax. The aim was to investigate the effects of Pue on the pathophysiology of DOR on a cellular level. It is hoped that findings from the present study may lay a foundation for its potential application in the clinical treatment of DOR.

\section{Materials and methods}

Animals. A total of 75 female specific-pathogen-free Sprague-Dawley rats (age, 4 weeks; weight, 60-70 g) were supplied by Beijing Vital River Laboratory Animal Technology Co., Ltd. [certificate no. SCXK (Beijing) 2017-0001]. The rats were housed in a temperature-controlled environment at $25^{\circ} \mathrm{C}$ and $50 \pm 10 \%$ humidity, with a 12 -h light/dark cycle and free access to a standard diet and water. The experiments and operations related to the animals involved in this study were performed with the approval of the Animal Ethics Committee of Guangdong Women and Children Hospital (Guangzhou, China).

Drugs, antibodies and reagents. The following drugs, reagents, antibodies and kits were used in the present study: Pue (Merck KGaA), VCD, sesame oil (Sigma-Aldrich; Merck KGaA), FSH ELISA kit (cat. no. E-EL-R0391c; Elabscience Biotechnology, Inc.), luteinizing hormone (LH) ELISA kit (cat.E-EL-H6019; Elabscience Biotechnology, Inc.), estradiol ( $\left.\mathrm{E}_{2}\right)$ kit (cat. no. KGE014; R\&D Systems, Inc.), Bcl-2 antibodies (cat. no. ab182858; Abcam), Bax antibodies (cat. no. ab216985; Abcam), caspase-3 antibodies (cat. no. ab32150; Abcam) and GAPDH antibodies (cat. no. ab8245; Abcam).

Instruments. The following instruments were used: CX21 light microscope (Olympus Corporation), EnSpire ${ }^{\circledR} 2300$ Enzyme Multimode Plate Reader (PerkinElmer, Inc.), MiniPROTEAN Tetra Cell and Trans-Blot SD Cell (Bio-Rad Laboratories, Inc.), PowerPac HC Electrophoresis system (Bio-Rad Laboratories, Inc.), TS-12F Automatic Biological Tissue Dehydrator (Xiaogan Hongye Medical Instrument Co., Ltd.) and a BMJ-M Pathological Tissue Embedding Machine and Embedding Freezer (Tianjin Tianli Aeronautical Mechanical and Electrical Co., Ltd.).

Establishment of the DOR animal model. After conducting a standard 7-day feeding period with the 4-week-old female Sprague Dawley rats, the rats were screened using smears of the vaginal exfoliated cells after opening the vulva, where the estrous cycle was found to be normal (Proestrus, a large number of oval nucleated epithelial cells and a small number of leukocytes; during estrus, a large number of a-nucleated keratinized epithelial cells; late estrus, non-nucleated keratinocytes, oval nucleated cells and leukocytes at the same time; estrous interval, a large number of white blood cells and a small number of nuclear epithelial cells). A total of 12 of the 60 rats (15 rats died due to the modeling process) were randomly selected for the normal group, whilst the remaining 48 rats were used in the model, Pue-L, Pue-M and Pue-H groups, with
12 rats in each group. Model, Pue-L, Pue-M and Pue-H groups were modeled by injection with VCD.

The feasibility of model establishment was mainly evaluated based on the following criteria: i) Observation of vaginal smears showed that the rats had a prolonged estrous cycle; ii) microscopic analysis revealing ovarian atrophy; iii) a decreased number of preantral and antral follicles; iv) intensified atresia; and v) significant hyperplasia of the ovarian stroma.

VCD was dissolved to a concentration of $80 \mathrm{mg} / \mathrm{kg}$ in sesame oil in preparation for injection, where an intraperitoneal injection was conducted once daily (starting at 10:00 a.m.) for 45 days, as described previously (15). Rats in the normal group were injected with an equal volume of sesame oil once a day. At $24 \mathrm{~h}$ after the last administration, all rats were anesthetized via an intraperitoneal injection of $1 \%$ pentobarbital sodium (40 mg/kg), following which blood from the inferior vena cava was collected $(8-10 \mathrm{ml})$. Subsequently, the anaesthetized rats were sacrificed by cervical dislocation before ovarian and uterus tissues were extracted after confirmation of cardiac arrest.

Grouping and administration. A total of 60 rats were randomly divided into the model, Pue-low dose (L) group (50 mg/kg), Pue-medium dose (M) group (100 mg/kg), Pue-high dose (H) group $(300 \mathrm{mg} / \mathrm{kg}$ ) and normal groups (17). The rats in each group were administered with Pue dissolved in saline by intragastric administration starting on the first day of modeling, then once a day for 45 days. The model and normal groups were administered with the same volume of saline once a day for the same period.

Sampling. The weight of the rats was measured and recorded 1 day before modeling, 1 week after modeling and at the end of modeling. Vena cava blood was drawn before the animals were sacrificed. The ovarian and uterus tissue samples were then obtained using the rapid aseptic method, where the wet weight was measured and the organ indices was calculated as follows: i) Ovary index = ovary mass / rat body weight; and ii) uterus index $=$ uterus mass / rat body weight. In total, $50 \%$ ovarian tissues from each rat was stored at $-70^{\circ} \mathrm{C}$, whilst the other $50 \%$ was fixed in $4 \%$ paraformaldehyde at room temperature for $48 \mathrm{~h}$ for subsequent use.

Ovarian tissue observation using $H \& E$ staining. Ovarian tissues were fixed in paraformaldehyde, progressively dehydrated with increasing concentrations of ethanol, embedded in paraffin, sectioned $(4 \mu \mathrm{m})$ and deparaffinized with ascending series of alcohol (70\% alcohol for $45 \mathrm{~min}, 75 \%$ alcohol for $45 \mathrm{~min}$ ). The sections were then stained with hematoxylin for $3 \mathrm{~min}$ and eosin for $20 \mathrm{sec}$ at room temperature, and visualized using an optical light microscope (magnification, x100).

ELISAs. After resting for $30 \mathrm{~min}$, the blood was centrifuged at $8000 \mathrm{x} \mathrm{g}$ and $4^{\circ} \mathrm{C}$ for $30 \mathrm{sec}$ before the serum was collected. Serum FSH, $\mathrm{LH}$ and $\mathrm{E}_{2}$ levels were measured using the respective $\mathrm{FSH}, \mathrm{LH}$ and $\mathrm{E}_{2}$ kits according to the manufacturers' protocols.

TUNEL staining of ovarian tissues. Paraffin-embedded tissue was cut into sections, placed in water and treated with 

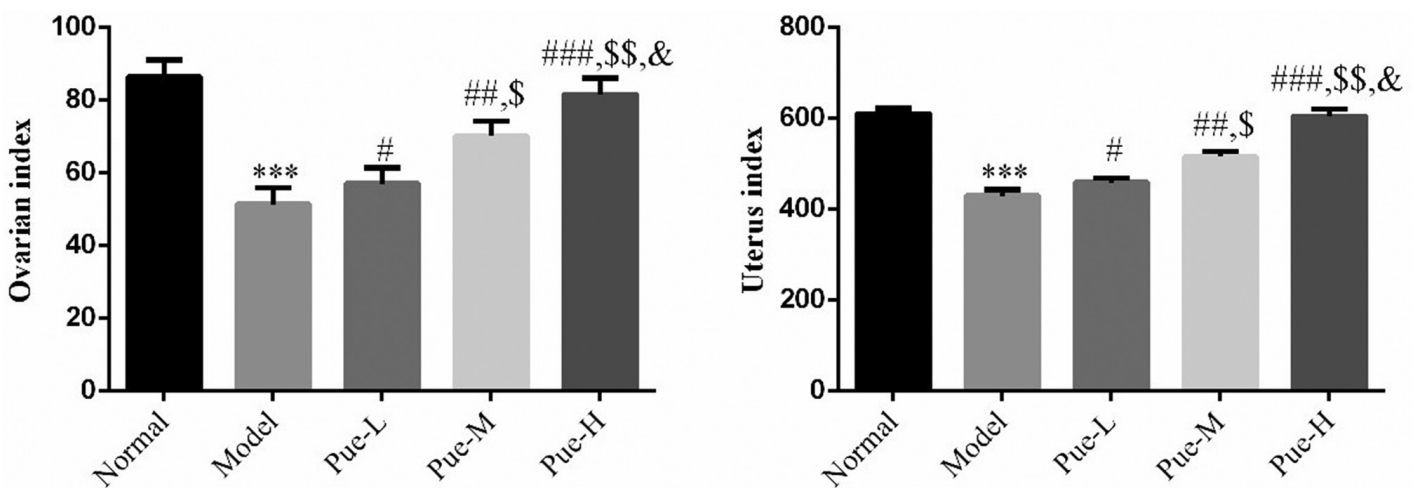

Figure 1. Effects of Pue on the ovarian and uterine indices of DOR model rats. Rats were divided into normal, model, Pue-L, Pue-M and Pue-H groups. ${ }^{* * * *} \mathrm{P}<0.001$ vs. normal; ${ }^{\# \mathrm{P}}<0.05,{ }^{\# \#} \mathrm{P}<0.01,{ }^{\# \# \#} \mathrm{P}<0.001$ vs. Model; ${ }^{~} \mathrm{P}<0.05,{ }^{\mathrm{S}} \mathrm{P}<0.01$ vs. Pue-L; ${ }^{\&} \mathrm{P}<0.05$ vs. Pue-M. DOR, diminished ovarian reserve; Pue, pueratin; L, low dose; $\mathrm{M}$, medium dose; $\mathrm{H}$, high dose.

a protease K (Sigma-Aldrich; Merck $\mathrm{KGaA}$ ) solution at $37^{\circ} \mathrm{C}$ for $15 \mathrm{~min}$. After washing three times with PBS for $3 \mathrm{~min}$, the TUNEL reaction mixture (including the TdT enzyme and dUTP marker solution) was added for $1 \mathrm{~h}$ in a humidified incubator at $37^{\circ} \mathrm{C}$, before being washed five times with PBS for 5 min. A confining liquid (cat. no. 4112APG; Richard Allan Scientific ${ }^{\mathrm{TM}}$; Thermo Fisher Scientific, Inc.) was then added and the samples were placed in a humidified incubator at $37^{\circ} \mathrm{C}$ for $30 \mathrm{~min}$. Positive and negative controls were included by adding deoxyribonuclease I reaction mixture and omitting the TdT enzyme reaction mixture, respectively. TUNEL positive cells were observed in $>4$ randomly selected fields under a fluorescent microscope (magnification, x200).

Immunohistochemical (IHC) staining of ovarian tissue. The 4- $\mu \mathrm{m}$ paraffin-embedded ovarian tissue sections after boiling in $0.1 \mathrm{~mol} / 1$ citric acid buffer solution ( $\mathrm{pH} 6.0$ ), antigen retrieval was performed at room temperature for $10 \mathrm{~min}$, followed by inactivation of endogenous catalase activity with $3 \% \mathrm{H}_{2} \mathrm{O}_{2}$ at room temperature for $5 \mathrm{~min}$. A Dako pen was used to mark the tissue site. Next, $\sim 50 \mu 110 \%$ normal donkey serum (cat. no. 017-000-001; Jackson ImmunoResearch Laboratories, Inc.) was added to the slides, which were incubated for $20 \mathrm{~min}$ at $37^{\circ} \mathrm{C}$. The serum on the slide was then removed and primary antibodies against Bcl-2 (1:200; cat. no. ab182858; Abcam), Bax (1:200; cat. no. ab216985; Abcam), caspase-3 (1:200; cat. no. ab32150; Abcam) and GAPDH (1:200; cat. no. ab8245; Abcam) were added dropwise. The diluent solution (PBS) without primary antibody was used as the negative control. The samples were then placed and incubated at $4^{\circ} \mathrm{C}$ overnight. Incubation with HRP-conjugated secondary antibodies (cat. no. BA1054; 1:2,000; Wuhan Boster Biological Technology, Ltd.) was performed at room temperature for $2 \mathrm{~h}$. DAB color development was then performed, including staining by hematoxylin at room temperature for $1 \mathrm{~h}$ and differentiation by hydrochloric acid ethanol. Progressive dehydration with increasing ethanol alcohol concentrations was then performed, followed by mounting in neutral balsam. The cells were observed and photographed under an optical light microscope (magnification, x200). Protein expression was analyzed using ImageJ v1.8.0.112 (National Institutes of Health).
Detection of Bcl-2 and Bax protein expression in the ovary using western blotting. The protein was extracted from rat ovarian tissues by RIPA lysis buffer (Beijing Solarbio Science \& Technology Co., Ltd.). Protein concentration was determined by a BCA protein assay kit according to the manufacturer's protocol and proteins ( $45 \mu \mathrm{g} / \mathrm{lane})$ were separated via $10 \%$ SDS-PAGE and transferred onto PVDF membranes. The membranes were subsequently blocked with 5\% BSA (Nanjing KeyGen Biotech Co., Ltd.) for $2 \mathrm{~h}$ at $4^{\circ} \mathrm{C}$, then incubated with Bcl-2 (1:1,000; cat. no. ab182858; Abcam), Bax (1:1000; cat. no. ab216985; Abcam), caspase-3 (1:1,000; cat. no. ab32150; Abcam) and GAPDH (1:1000; cat. no. ab8245; Abcam) rabbit anti-rat polyclonal antibodies overnight at $4{ }^{\circ} \mathrm{C}$. Following the primary antibody incubation, the membrane was washed and incubated with a HRP-conjugated goat anti-rabbit secondary antibody (cat. no. BA1054; 1:2,000; Wuhan Boster Biological Technology, Ltd.) at $37^{\circ} \mathrm{C}$ for $2 \mathrm{~h}$. The blots were then incubated with WesternBright ECL (APG Bio, Ltd.). GAPDH was used as the internal reference. The results were analyzed using ImageJ version 1.47 (National Institutes of Health).

Statistical analysis. SPSS 22.0 (IBM Corp.) was used for statistical analysis. The results are presented as the mean $\pm \mathrm{SD}$ from 12 rats per group. One way ANOVA was used for comparison between multiple groups, followed by Tukey's tests. $\mathrm{P}<0.05$ was considered to indicate a statistically significant difference.

\section{Results}

Effects of Pue on rat ovarian and uterine indices in the DOR model. Compared with those in the normal group, the ovarian and uterine indices in the model group were significantly decreased (two rats died during the experiment due to injection injury; $\mathrm{P}<0.001$; Fig. 1), suggesting that the ovaries and uteri of rats in the DOR model were atrophied. Compared with those in the model group, the ovarian and uterine indices in the Pue intervention group were significantly increased (one rat died during the experiment due to injection injury; $\mathrm{P}<0.05$; Fig. 1) in a dose-dependent manner $(\mathrm{P}<0.05$; Fig. 1). This suggests that the extent of ovary and uterus atrophy was at least partially reduced in rats following Pue treatment. 

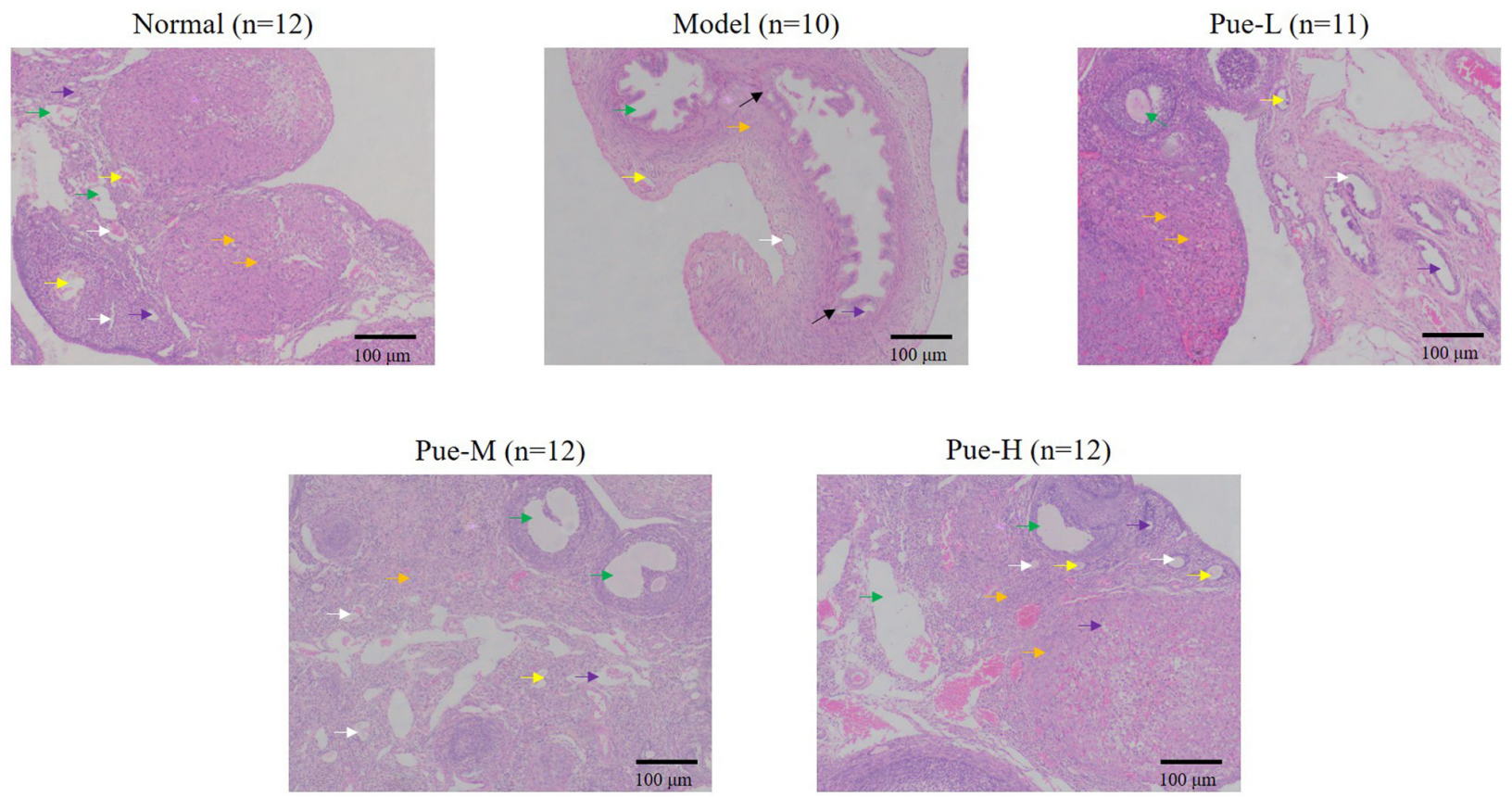

Figure 2. Effects of Pue on the morphology of ovaries in DOR model rats. Rats were divided into normal, model, Pue-L, Pue-M and Pue-H groups. Scale bars, $100 \mu \mathrm{m}$. White arrow, primordial follicles; yellow arrow, primary follicles; green arrow, mature follicles; violet arrow, oocytes; orange arrow, granulosa cells . DOR, diminished ovarian reserve; Pue, pueratin; L, low dose; M, medium dose; H, high dose.

\section{Effect of Pue on the morphology of ovaries of rats in the DOR} model

Macroscopic observations. Compared with that in the normal group, the volume of the ovary was observed to be markedly decreased after modeling, where there were different degrees of adhesion to the surrounding tissues (Fig. 2; black arrow).

Microscopic findings. Compared with that in the normal group, the number of rat ovarian primordial and primary follicles in the model group was markedly decreased. It was also found indirectly that the number of mature follicles was also decreased. The size of oocytes was reduced, the number of granulosa cells was decreased and the arrangement became disordered. However, after intragastric Pue administration, the pathology of the ovary was markedly improved, for example, the number of follicles were higher in the Pue groups compared with the model group, suggesting a curative relationship (Fig. 2).

Effects of Pue on the apoptosis of rat ovarian cells in the DOR model. TUNEL assay results showed that, compared with that in the normal group, the number of apoptotic cells in the ovaries of the model group was significantly increased $(\mathrm{P}<0.001$; Fig. 3). After intervention with Pue, compared with that in the model group, the number of apoptotic cells in the ovarian tissue of the Pue groups was significantly decreased $(\mathrm{P}<0.05$; Fig. 3). These significant reductions in the number of apoptotic cells in the ovarian tissue among Pue groups were also found to be dose-dependent $(\mathrm{P}<0.05$; Fig. 3).

Effects of Pue on rat serum FSH, LH and $E_{2}$ levels in the DOR model. Compared with those in the normal group, FSH, LH and $\mathrm{E}_{2}$ levels in the model group were significantly changed $(\mathrm{P}<0.001$; Fig. 4). Specifically, FSH and LH levels were significantly increased, whilst $\mathrm{E}_{2}$ levels were significantly decreased. After intervention with Pue, compared with those in the model group, the levels of FSH and LH in each Pue group were significantly decreased, whereas and the level of $\mathrm{E}_{2}$ was significantly increased $(\mathrm{P}<0.05 ; \mathrm{Fig} .4)$. In addition, there was a significant dose-effect relationship among the Pue groups $(\mathrm{P}<0.05$; Fig. 4).

Apoptotic caspase-3 protein expression analysis using IHC and WB. Compared with that in the normal group, the expression of caspase- 3 protein in the model group was significantly upregulated ( $\mathrm{P}<0.001$; Fig. 5A and B). However, following Pue administration, the protein expression levels of caspase- 3 were significantly downregulated compared with that the model group in a dose-dependent manner $(\mathrm{P}<0.05$; Fig. 5A and $\mathrm{B})$.

Detection of the expression levels of Bcl-2 and Bax proteins in the epithelial regions of ovarian tissues using IHC. Compared with that in the normal group, the expression levels of Bcl-2 in the model group tissues were decreased, whilst that of Bax was significantly increased $(\mathrm{P}<0.001$; Fig. 6A and $\mathrm{B})$, suggesting that the high expression of Bax protein and the low expression of $\mathrm{Bcl}-2$ protein in the model group may accelerate the apoptosis of follicles and result in the decline in ovarian reserve. Compared with that in the model group, the expression of Bcl-2 protein in the ovaries of the Pue intervention group was significantly increased, whilst the expression of Bax protein was significantly decreased $(\mathrm{P}<0.05$; Fig. 6A and $\mathrm{B})$. In addition, there was a significant dose-dependent effect on the expression of Bcl-2 and Bax among the Pue groups $(\mathrm{P}<0.05$; Fig. 5A and B).

Detection of the expression levels of Bcl-2 and Bax proteins in ovarian tissues using WB. Compared with that in the normal 
Normal $(n=12)$

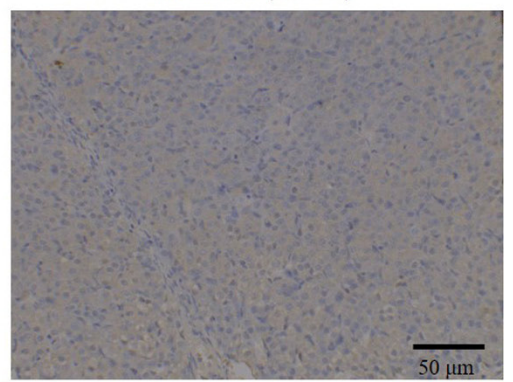

Pue-M ( $=12)$

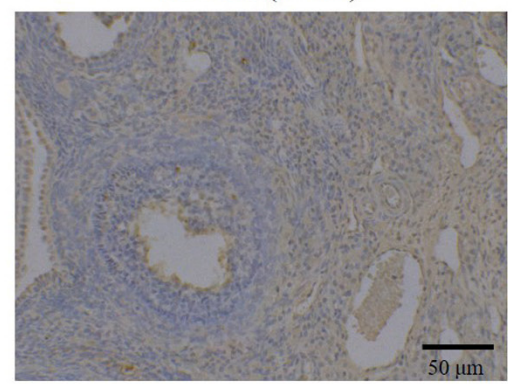

Model $(\mathrm{n}=10)$

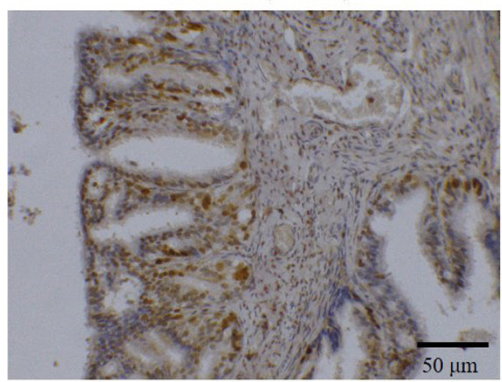

Pue-H (n=12)

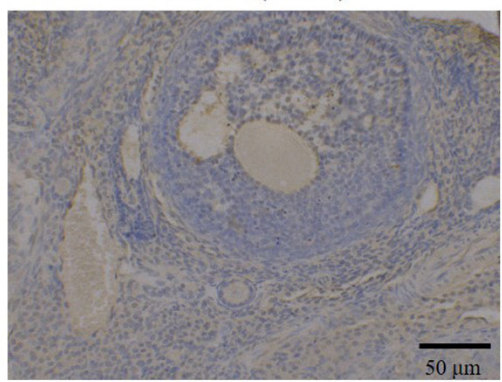

Pue-L $(n=11)$
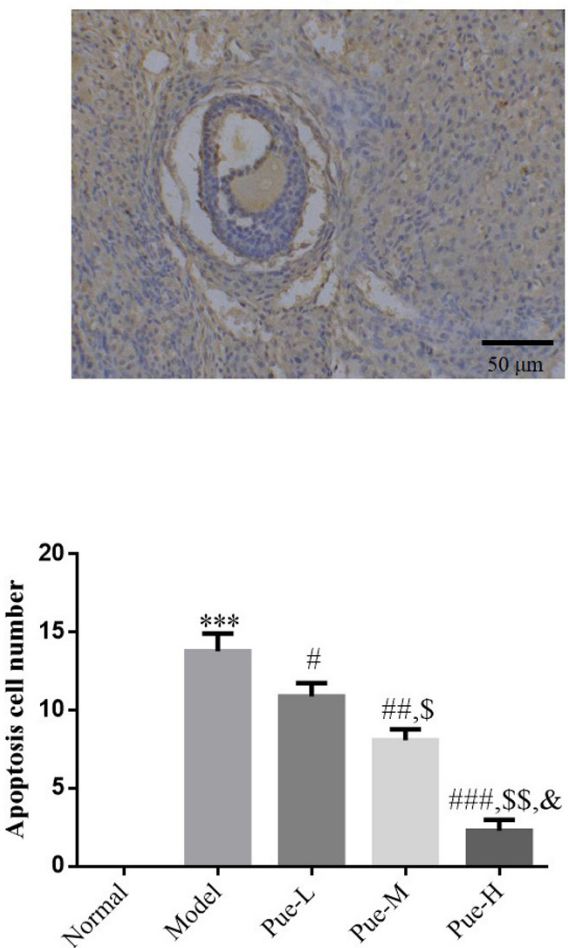

Figure 3. Apoptotic cell number in the different treatment groups. Rats were divided into normal, model, Pue-L, Pue-M and Pue-H groups. Scale bars, $50 \mu \mathrm{m}$. ${ }^{* * * *} \mathrm{P}<0.001$ vs. normal; ${ }^{\# \mathrm{P}}<0.05,{ }^{\# \#} \mathrm{P}<0.01,{ }^{\# \# \#} \mathrm{P}<0.001$ vs. model; ${ }^{\$} \mathrm{P}<0.05,{ }^{\$ \$} \mathrm{P}<0.01$ vs. Pue-L; ${ }^{\&} \mathrm{P}<0.05$ vs. Pue-M. DOR, diminished ovarian reserve; Pue, pueratin; $\mathrm{L}$, low dose; $\mathrm{M}$, medium dose; $\mathrm{H}$, high dose.
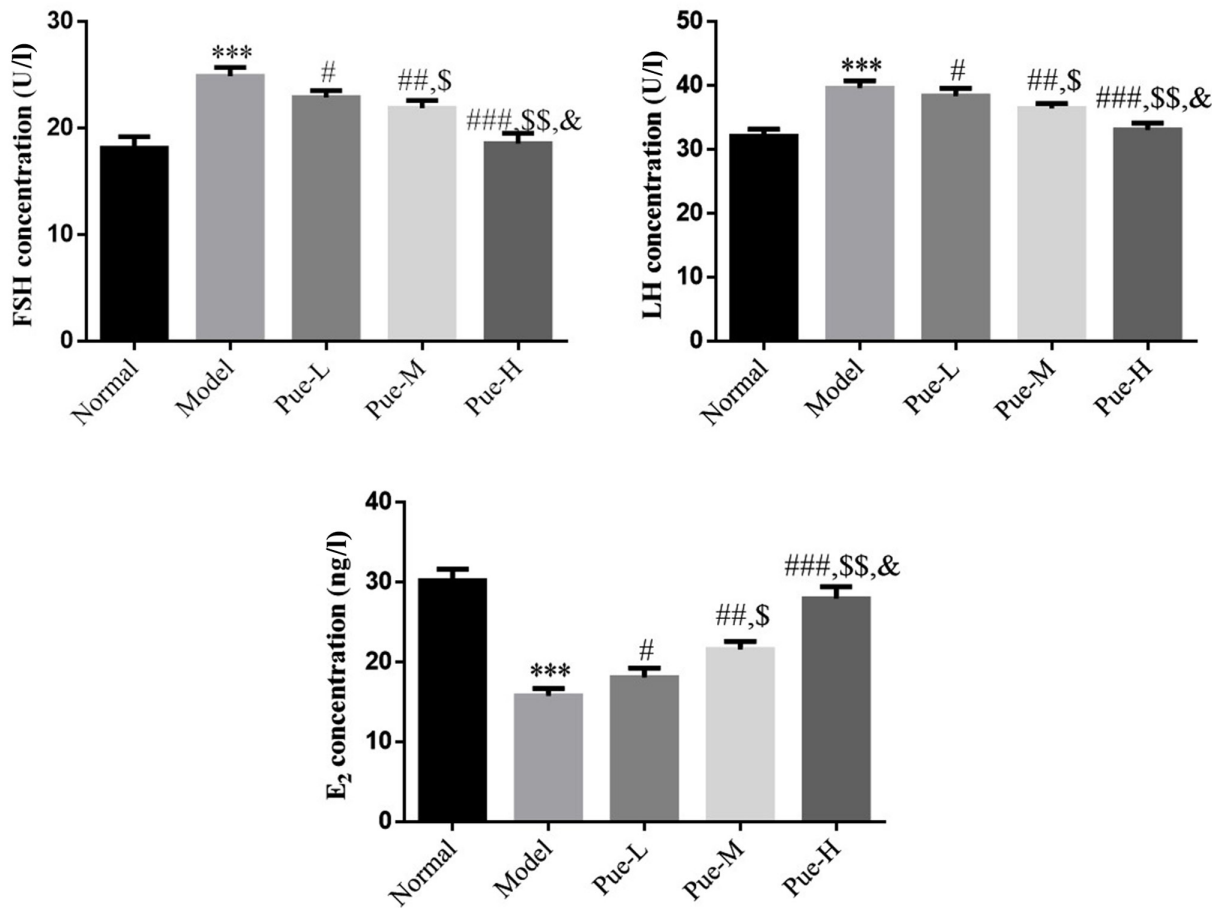

Figure 4. Effects of Pue on FSH, LH and $\mathrm{E}_{2}$ levels in the serum of DOR model rats. Rats were divided into normal, model, Pue-L, Pue-M and Pue-H groups. ${ }^{* * * *} \mathrm{P}<0.001$ vs. normal; ${ }^{\#} \mathrm{P}<0.05,{ }^{\# \#} \mathrm{P}<0.01$ and ${ }^{\# \# \#} \mathrm{P}<0.001$ vs. model; ${ }^{\$} \mathrm{P}<0.05$ and ${ }^{\$ \$} \mathrm{P}<0.01$ vs. Pue-L; ${ }^{\&} \mathrm{P}<0.05$ vs. Pue-M. DOR, diminished ovarian reserve; Pue, pueratin; L, low dose; M, medium dose; H, high dose; FSH, follicle-stimulating hormone; LH, luteinizing hormone; $\mathrm{E}_{2}$, estradiol.

group, the expression of Bcl-2 protein in the model group was significantly decreased whereas the expression of Bax was significantly increased $(\mathrm{P}<0.001 ;$ Fig. 7$)$. In addition, compared with that in the model group, there was a significant upregulation in the Bcl-2/Bax ratio $(\mathrm{P}<0.001$; Fig. 7), suggesting that the high expression of Bax protein and the low expression of 
A
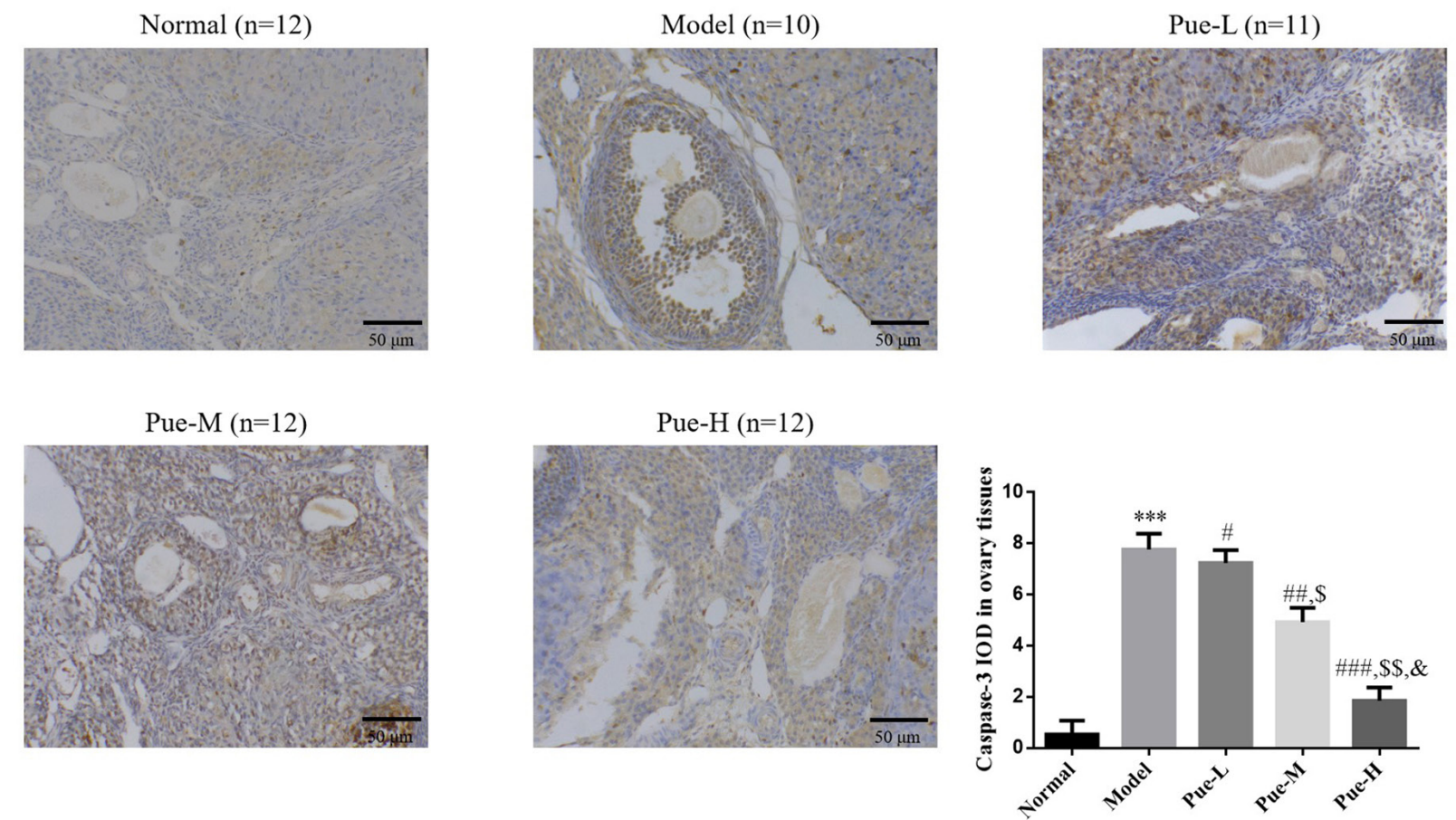

B
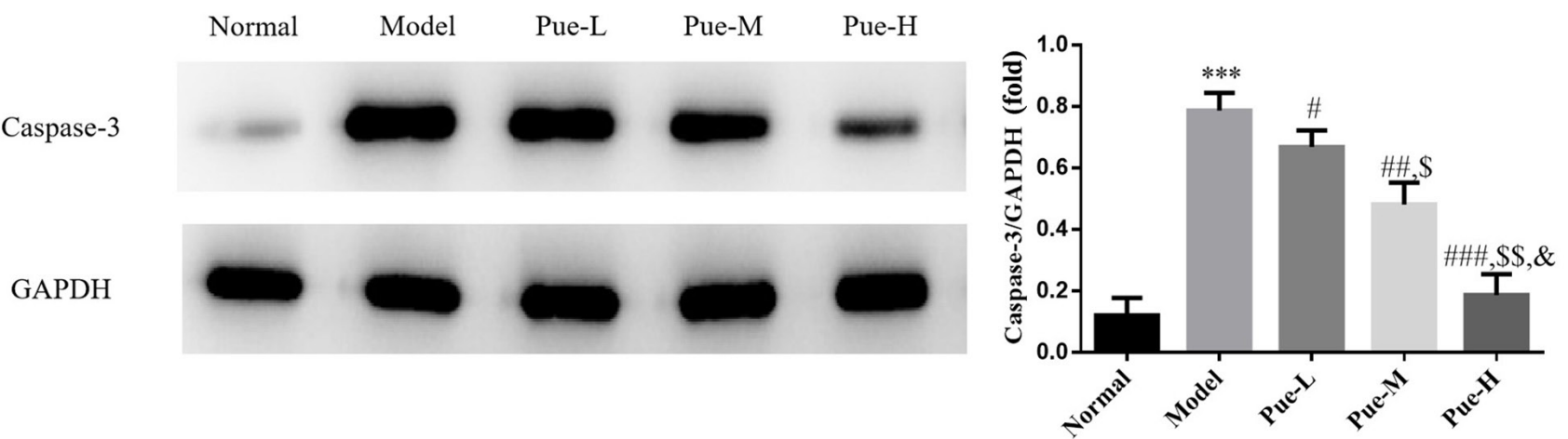

Figure 5. Caspase-3 protein expression in ovaries as determined using IHC and WB. Rats were divided into normal, model, Pue-L, Pue-M and Pue-H groups (A) Caspase-3 protein expression was analyzed using IHC assays. Scale bars, $50 \mu \mathrm{m}$. (B) Caspase-3 protein expression was measured using WB assays ${ }^{* * * *} \mathrm{P}<0.001$ vs. normal; ${ }^{\# \mathrm{P}}<0.05,{ }^{\# \#} \mathrm{P}<0.01,{ }^{\# \#} \mathrm{P}<0.001$ vs. model; ${ }^{\$} \mathrm{P}<0.05,{ }^{\$ \$} \mathrm{P}<0.01$ vs. Pue-L; ${ }^{\&} \mathrm{P}<0.05$ vs. Pue-M. DOR, diminished ovarian reserve; Pue, pueratin; L, low dose; M, medium dose; H, high dose; IHC, immunohistochemistry; WB, western blotting; IOD, integrated optical density.

Bcl-2 protein in the model group may accelerate the apoptosis of follicles and lead to the decline in ovarian reserve. Compared with that in the model group, the protein expression of Bcl-2 in the ovaries of the Pue intervention groups were significantly increased $(\mathrm{P}<0.05$; Fig. 7$)$, whereas that of Bax was significantly decreased ( $\mathrm{P}<0.05 ;$ Fig. 7$)$. Accordingly, the $\mathrm{Bcl}-2 / \mathrm{Bax}$ ratio was also significantly increased in the Pue intervention groups compared with that in the model group, where a dose-dependent effect was observed $(\mathrm{P}<0.05$; Fig. 7).

\section{Discussion}

As early as the fetal stage, most human ovaries have follicles (17). After 6-8 weeks of embryonic development, mitosis occurs in the primordial germ cells, where the number and volume of cells increases (18). Following development, the number of oogonia is $\sim 600,000$ (19). Following growth of the fetus for 11-12 weeks, oogonia become oocytes through meiosis (20). After 16-20 weeks, the number of germ cells reaches its peak at 6-7 million, where oocytes account for $\sim 33 \%$ and primary oocytes account for $\sim 66 \%$ (20). As the fetus continues to develop, the number of cells decreases gradually, such that by 40 weeks of gestation, $>66 \%$ of the oocytes die due to apoptosis and the number remaining is reduced to $\sim 2$ million oocytes (21). Primary oocytes are normally surrounded by granulosa cells to form the primordial follicle, which is the basic reproductive unit of women and the only form of oocyte reserve (12). Numerous ovarian follicles die after birth (22). Follicular atresia is the gradual degradation of ovarian follicles at all stages of development (23). By adolescence, typically 
A

Normal $(n=12)$

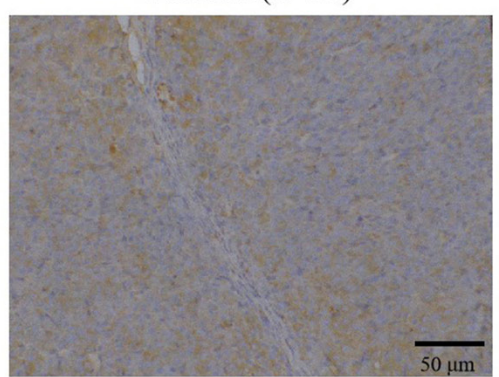

Pue-M (n=12)

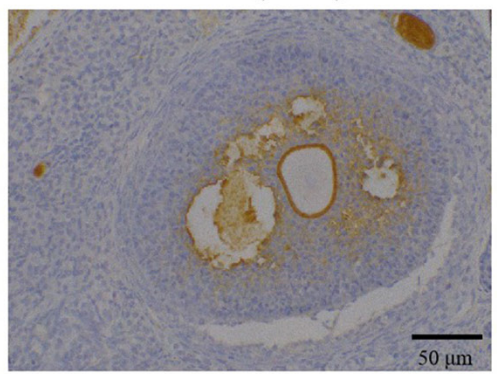

B

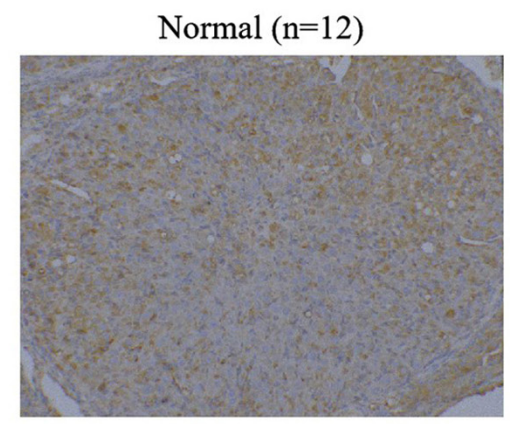

Pue-M (n=12)

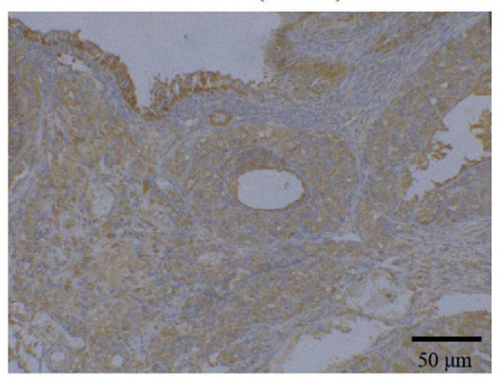

Model ( $\mathrm{n}=10)$

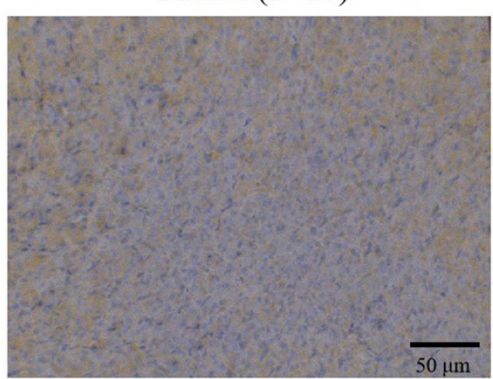

Pue-H $(n=12)$
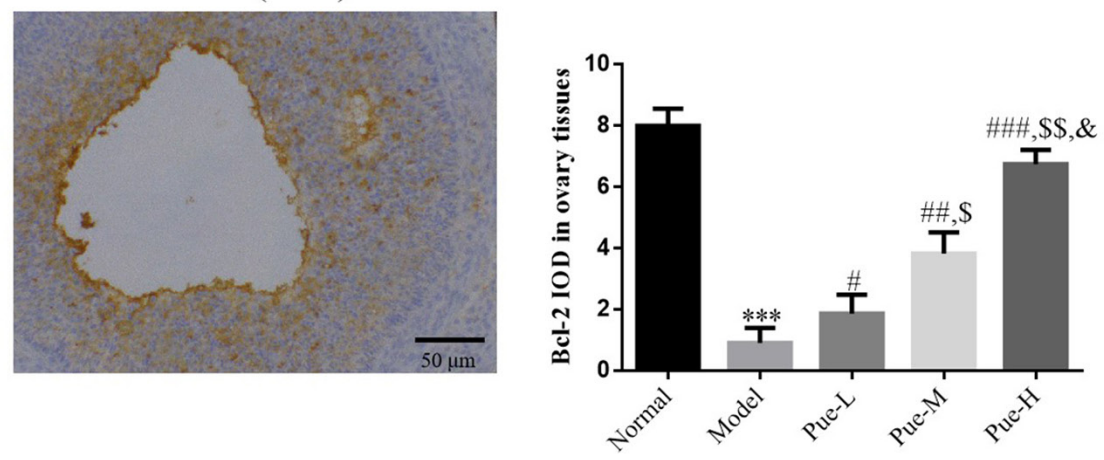

Model $(n=10)$

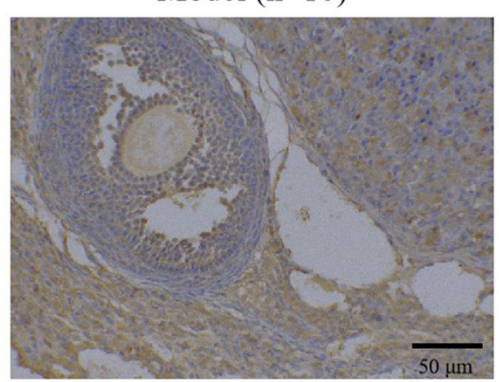

Pue-H (n=12)

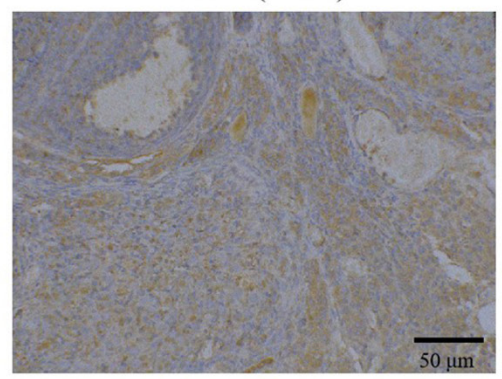

Pue-L (n=11)

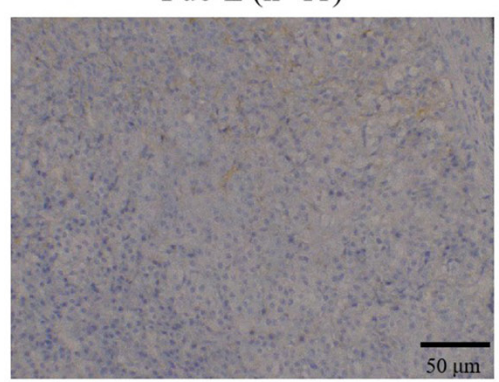

$\mu \mathrm{m}$ 


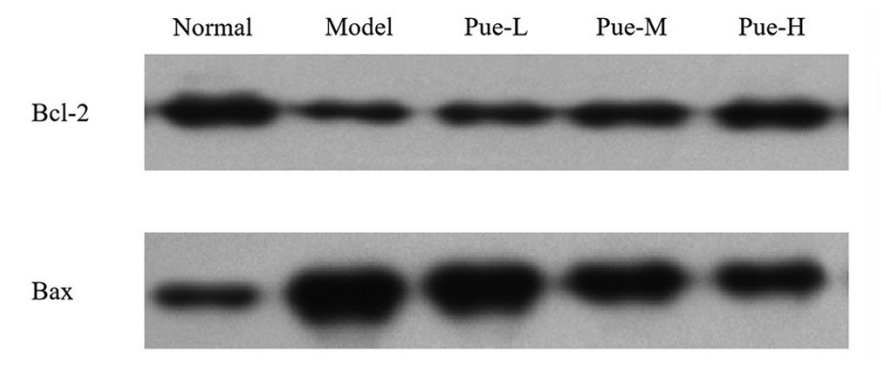

GAPDH
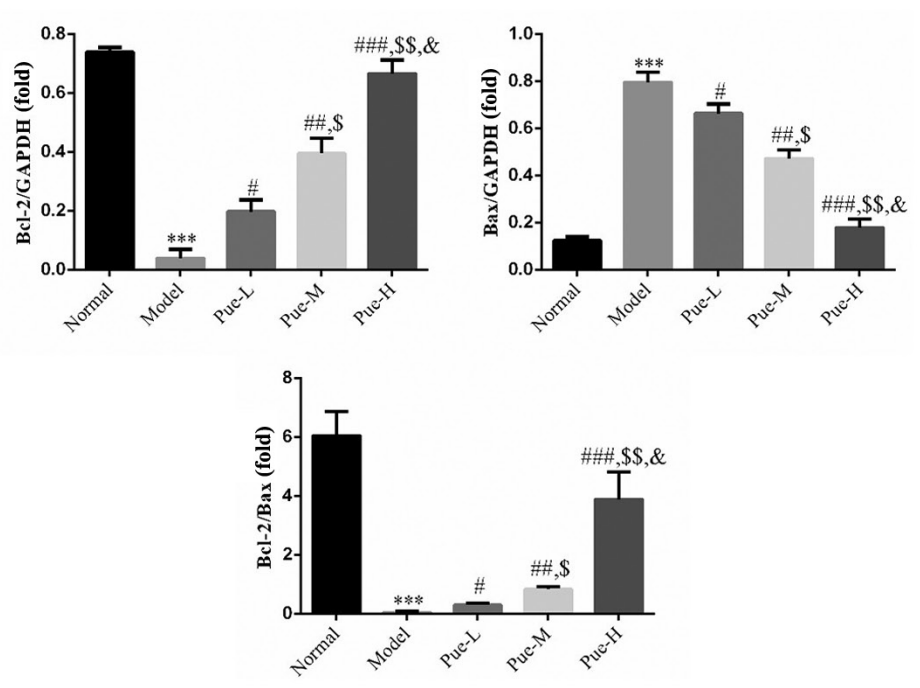

Figure 7. Bcl-2 and Bax protein expression as determined using western blotting. Rats were divided into normal, model, Pue-L, Pue-M and Pue-H groups. ${ }^{* * * *} \mathrm{P}<0.001$ vs. normal; ${ }^{\#} \mathrm{P}<0.05,{ }^{\# \#} \mathrm{P}<0.01,{ }^{\# \# \#} \mathrm{P}<0.001$ vs. model; ${ }^{\$} \mathrm{P}<0.05,{ }^{\$ \$} \mathrm{P}<0.01$ vs. Pue-L; ${ }^{\&} \mathrm{P}<0.05$ vs. Pue-M. DOR, diminished ovarian reserve; Pue, pueratin; L, low dose; M, medium dose.

required for follicular development and maturation depend on the proliferation and differentiation states of granulosa cells in the follicles, where apoptosis of granulosa cells is a prerequisite for follicular atresia (27). This directly affects the quantity and quality of follicles.

The pathophysiology of DOR is complex, where its etiology remains unclear. The generally accepted mechanism of DOR include follicular atresia caused by the rapid depletion of oocytes, abnormal proliferation and low differentiation of granulosa cells or the apoptosis of granulosa cells (28-30). The aim of the present study was to evaluate the atresia of primordial and primary follicles and the associated apoptosis of ovarian granulosa cells. Therefore, an animal model of DOR was established using the toxic chemical VCD. VCD was previously found to accelerate the natural apoptosis of follicles (31), where primary follicles are the main targets of VCD in the ovary. Abnormal VCD secretion is associated with a reduction in the number of viable follicles in the ovaries of patients with DOR (32). In the present study, the results of modeling were evaluated using vaginal smears, which suggested that VCD treatment was ideal for the study of DOR in animal models. Results from the present study revealed that apoptosis of oocytes and granulosa cells in the follicles was increased, resulting in the increase of follicular atresia and reduction of the ovarian reserve. Therefore, these results suggest that the number of primordial follicles and the number of viable follicles in the model group were lower than those in the normal group. In addition, since the apoptosis rate of ovarian granulosa cells in the model group was significantly higher compared with that in the normal group, establishment of the DOR rat model appears to be successful.

Apoptosis is the process of self-destruction that occurs in both normal physiological conditions and in disease states (33). Under normal conditions, the balance between granulosa cell mitosis and apoptosis is synchronized (34). When the apoptosis rate of granulosa cells reaches $>10 \%$, follicular atresia occurs (4), suggesting that apoptosis serves as the key event during follicular atresia. Since the number of primordial follicles in the ovary is fixed at birth, follicular atresia serves an important physiological function in the ovary, since this involves the removal of redundant tissues (35). Therefore, atresia of a small number of follicles does not normally affect the normal development of surrounding follicles (26). By contrast, DOR occurs when the speed of atresia in a large number of follicles is faster than the physiological metabolic rate (36).

Bcl-2 and Bax proteins are members of the Bcl-2 family of proteins, which can be sub-divided into the following two categories: i) Proteins represented by Bcl-2, which inhibit apoptosis; and ii) proteins represented by Bax, which promote apoptosis (37-39). Results from the present study suggested that VCD treatment led to an increase in FSH and LH levels, a decrease in the ovarian secretion of $\mathrm{E}_{2}$, a reduction in Bcl-2 expression and an elevation in Bax expression, which ultimately promoted the apoptosis of follicular granulosa cells. As pro-caspase 3 does not accurately reflect apoptotic activity, this may reflect another limitation of the present study. FSH and $\mathrm{LH}$ regulate the apoptosis and secretion of ovarian granulosa cells, which serve an important role in the regulation of follicular development and ovulation (28). The present study demonstrates that Pue may inhibit the apoptosis of follicles by downregulating FSH and LH levels whilst upregulating $E_{2}$ levels, in addition to upregulating the expression levels of Bcl-2 protein whilst downregulating the expression of Bax protein. The purpose of the present study was to test the effects of Pue on the pathophysiology and Bax and Bcl-2 expression in rats with DOR. However, there are limitations in the present study, as it remains unclear what the correlation is among Bcl-2, Bax expression and hormone levels during DOR.

In conclusion, the present study demonstrated that Pue treatment downregulated FSH and LH levels, stimulated $\mathrm{E}_{2}$ secretion and regulated the expression of the Bcl-2 family of proteins, Bcl-2 and Bax. By upregulating the expression of Bcl-2 protein and downregulating the expression of Bax protein, Pue was observed to inhibit apoptosis to preserve ovarian reserves, which may prove to be useful for the clinical prevention and treatment of DOR. 


\section{Acknowledgements}

Not applicable.

\section{Funding}

The present study was supported by the Natural Science Foundation of Guangdong Province (grant no. 2016A030310318).

\section{Availability of data and materials}

The datasets used and/or analyzed during the current study are available from the corresponding author on reasonable request.

\section{Authors' contributions}

HW drafted the manuscript. QQ conceived the study and participated in the manuscript preparation. HW, XZ, YC and LY assisted in the literature search and edited the manuscript. YC revised the manuscript and checked the data of our experiment. HW, XZ, YC and LY performed the experiment. QQ and YC confirm the authenticity of all the raw data and checked the grammar in manuscript. All authors have read and approved the final manuscript.

\section{Ethics approval and consent to participate}

The experiments and operations related to the animals involved in this study were performed with the approval of the Animal Ethics Committee of Guangdong Women and Children Hospital (Guangzhou, China).

\section{Patient consent for publication}

Not applicable.

\section{Competing interests}

The authors declare that they have no competing interests.

\section{References}

1. Jaillard S, Sreenivasan R, Beaumont M, Robevska G, Dubourg C Knarston IM, Akloul L, van den Bergen J, Odent S, Croft B, et al: Analysis of NR5A1 in 142 patients with premature ovarian insufficiency, diminished ovarian reserve, or unexplained infertility. Maturitas 131: 78-86, 2020

2. Tanaka Y, Hsueh AJ and Kawamura K: Surgical approaches of drug-free in vitro activation and laparoscopic ovarian incision to treat patients with ovarian infertility. Fertil Steril 114: 1355-1357, 2020.

3. Kawamura K, Kawamura N and Hsueh AJ: Activation of dormant follicles: A new treatment for premature ovarian failure? Curr Opin Obstet Gynecol 28: 217-222, 2016.

4. Gleicher N and Barad DH: Dehydroepiandrosterone (DHEA) supplementation in diminished ovarian reserve (DOR). Reprod Biol Endocrinol 9: 67, 2011.

5. Quaas AM and Legro RS: Pharmacology of medications used for ovarian stimulation. Best Pract Res Clin Endocrinol Metab 33 : $21-33,2019$

6. Cohen J, Chabbert-Buffet N and Darai E: Diminished ovarian reserve, premature ovarian failure, poor ovarian responder - a plea for universal definitions. J Assist Reprod Genet 32: 1709-1712, 2015.
7. Chen C, Li S, Hu C, Cao W, Fu Q, Li J, Zheng L and Huang J: Protective Effects of Puerarin on Premature Ovarian Failure via Regulation of Wnt/ $\beta$-catenin Signaling Pathway and Oxidative Stress. Reprod Sci 28: 982-990, 2021.

8. Chlebowski RT, Manson JE, Anderson GL, Cauley JA, Aragaki AK, Stefanick ML, Lane DS, Johnson KC, Wactawski-Wende J, Chen C, et al: Estrogen plus progestin and breast cancer incidence and mortality in the Women's Health Initiative Observational Study. J Natl Cancer Inst 105: 526-535, 2013.

9. Worsley R, Davis SR, Gavrilidis E, Gibbs Z, Lee S, Burger H and Kulkarni J: Hormonal therapies for new onset and relapsed depression during perimenopause. Maturitas 73: 127-133, 2012.

10. Zhou YX, Zhang H and Peng C: Puerarin: A review of pharmacological effects. Phytother Res 28: 961-975, 2014.

11. Jiang M, Yun Q, Niu G, Gao Y, Shi F and Yu S: Puerarin prevents inflammation and apoptosis in the neurocytes of a murine Parkinson's disease model. Genet Mol Res: Oct 5, 2016 (Epub ahead of print).doi: $10.4238 / \mathrm{gmr} .15047501$.

12. Zhou X, Bai C, Sun X, Gong X, Yang Y, Chen C, Shan G and Yao Q: Puerarin attenuates renal fibrosis by reducing oxidative stress induced-epithelial cell apoptosis via MAPK signal pathways in vivo and in vitro. Ren Fail 39: 423-431, 2017.

13. Xue Q, Liu Y, He R, Yang S, Tong J, Li X, Chen Y and $\mathrm{Xu}$ X: Lyophilized Powder of Catalpol and Puerarin Protects Neurovascular Unit from Stroke. Int J Biol Sci 12: 367-380, 2016.

14. Ma Y, Gai Y, Yan J, Li J and Zhang Y: Puerarin Attenuates Anoxia/Reoxygenation Injury Through Enhancing Bcl-2 Associated Athanogene 3 Expression, a Modulator of Apoptosis and Autophagy. Med Sci Monit 22: 977-983, 2016.

15. Gaumer S, Guénal I, Brun S, Théodore L and Mignotte B: Bcl-2 and Bax mammalian regulators of apoptosis are functional in Drosophila. Cell Death Differ 7: 804-814, 2000.

16. 16. Lin H, Zhang XM, Chen C and Chen BD: Apoptosis of Mo7e leukemia cells is associated with the cleavage of Bcl-2 into a shortened fragment that is not functional for heterodimerization with Bcl-2 and Bax. Exp Cell Res 261: 180-186, 2000.

17. Li L, Dong J, Yan L, Yong J, Liu X, Hu Y, Fan X, Wu X, Guo H, Wang X, et al: Single-Cell RNA-Seq Analysis Maps Development of Human Germline Cells and Gonadal Niche Interactions. Cell Stem Cell 20: 858-873.e4, 2017.

18. Xu M, Che L, Yang Z, Zhang P, Shi J, Li J, Lin Y, Fang Z, Che L, Feng B, et al: Proteomic Analysis of Fetal Ovaries Reveals That Primordial Follicle Formation and Transition Are Differentially Regulated. BioMed Res Int 2017: 6972030, 2017.

19. Birt JA, Taylor KH, Davis JW and Sharpe-Timms KL: Developmental exposure of fetal ovaries and fetal germ cells to endometriosis in an endometriosis model causes differential gene expression in the preimplantation embryos of the first-generation and second-generation embryos. Fertil Steril 100: 1436-1443, 2013.

20. Liu H, Zhang X, Zhong X, Li Z, Cai S, Yang P, Ou C and Chen M: Puerarin inhibits vascular calcification of uremic rats. Eur J Pharmacol 855: 235-243, 2019.

21. Lei L, Zhang H, Jin S, Wang F, Fu M, Wang H and Xia G: Stage-specific germ-somatic cell interaction directs the primordial folliculogenesis in mouse fetal ovaries. J Cell Physiol 208: 640-647, 2006.

22. Juneja SC, Barr KJ, Enders GC and Kidder GM: Defects in the germ line and gonads of mice lacking connexin43. Biol Reprod 60: 1263-1270, 1999.

23. Pepling ME: From primordial germ cell to primordial follicle: Mammalian female germ cell development. Genesis 44: 622-632, 2006.

24. Vaskivuo TE and Tapanainen JS: Apoptosis in the human ovary. Reprod Biomed Online 6: 24-35, 2003.

25. Perez GI, Maravei DV, Trbovich AM, Cidlowski JA, Tilly JL and Hughes FM Jr: Identification of potassium-dependent and -independent components of the apoptotic machinery in mouse ovarian germ cells and granulosa cells. Biol Reprod 63: 1358-1369, 2000.

26. Wang S, Sun M, Yu L, Wang Y, Yao Y and Wang D: Niacin Inhibits Apoptosis and Rescues Premature Ovarian Failure. Cell Physiol Biochem 50: 2060-2070, 2018.

27. Silva JRV, Lima FEO, Souza ALP and Silva AWB: Interleukin-1 $\beta$ and TNF- $\alpha$ systems in ovarian follicles and their roles during follicular development, oocyte maturation and ovulation. Zygote 28: 270-277, 2020.

28. Wang S, Lin S, Zhu M, Li C, Chen S, Pu L, Lin J, Cao L and Zhang Y: Acupuncture Reduces Apoptosis of Granulosa Cells in Rats with Premature Ovarian Failure Via Restoring the PI3K/Akt Signaling Pathway. Int J Mol Sci 20: E6311, 2019. 
29. Kappeler CJ and Hoyer PB: 4-vinylcyclohexene diepoxide: A model chemical for ovotoxicity. Syst Biol Reprod Med 58: 57-62, 2012.

30. Chauhan P, Sodhi A and Tarang S: Cisplatin-treated murine peritoneal macrophages induce apoptosis in L929 cells: Role of Fas-Fas ligand and tumor necrosis factor-tumor necrosis factor receptor 1. Anticancer Drugs 18: 187-196, 2007.

31. Almarzoug MHA, Ali D, Alarifi S, Alkahtani S and Alhadheq AM: Platinum nanoparticles induced genotoxicity and apoptotic activity in human normal and cancer hepatic cells via oxidative stress-mediated $\mathrm{Bax} / \mathrm{Bcl}-2$ and caspase-3 expression. Environ Toxicol 35: 930-941, 2020.

32. Roosa KA, Mukai M and Place NJ: 4-Vinylcyclohexene diepoxide reduces fertility in female Siberian hamsters when treated during their reproductively active and quiescent states. Reprod Toxicol 51: 40-46, 2015.

33. Elmore S: Apoptosis: A review of programmed cell death. Toxicol Pathol 35: 495-516, 2007.

34. Luciano AM, Modina S, Gandolfi F, Lauria A and Armstrong DT: Effect of cell-to-cell contact on in vitro deoxyribonucleic acid synthesis and apoptosis responses of bovine granulosa cells to insulin-like growth factor-I and epidermal growth factor. Biol Reprod 63: 1580-1585, 2000.

35. Gosden RG and Faddy MJ: Biological bases of premature ovarian failure. Reprod Fertil Dev 10: 73-78, 1998.
36. Klein JL, Adams SM, De Moura AF, Alves Filho DC, Maidana FM, Brondani IL, Cocco JM, Rodrigues LDS, Pizzuti LAD and Da Silva MB: Productive performance of beef cows subjected to different nutritional levels in the third trimester of gestation. Animal 15: 100089, 2021.

37. Wang $\mathrm{H}$, Jiao $\mathrm{H}$, Jiang $\mathrm{Z}$ and Chen R: Propofol inhibits migration and induces apoptosis of pancreatic cancer PANC-1 cells through miR-34a-mediated E-cadherin and LOC285194 signals. Bioengineered 11: 510-521, 2020.

38. Erfani S, Moghimi A, Aboutaleb N and Khaksari M: Protective Effects of Nucleobinding-2 After Cerebral Ischemia Via Modulating Bcl-2/Bax Ratio and Reducing Glial Fibrillary Acid Protein Expression. Basic Clin Neurosci 10: 451-459, 2019.

39. Fakhrabadi HG, Rabbani-Chadegani A, Ghadam P and Amiri S: Protective effect of bleomycin on 5-azacitidine induced cytotoxicity and apoptosis in mice hematopoietic stem cells via $\mathrm{Bcl}-2 / \mathrm{Bax}$ and $\mathrm{HMGB} 1$ signaling pathway. Toxicol Appl Pharmacol 396: 114996, 2020.

This work is licensed under a Creative Commons Attribution-NonCommercial-NoDerivatives 4.0 International (CC BY-NC-ND 4.0) License. 